\title{
Electrostatic surface potential decay on cellulose acetate
}

\author{
E. Borzabadi ${ }^{1}$, A. S.Vaughan ${ }^{2}$ and A. G. Bailey ${ }^{2}$ \\ ${ }^{1}$ University of Isfahan, Isfahan, Iran \\ ${ }^{2}$ University of Southampton, Southampton, UK
}

\begin{abstract}
The decay of surface potential on cellulose acetate has been studied as a function of appropriate parameters. This material was chosen because of the relatively rapid nature of the decay process and the well-known interactions that occur between it and its environment. It was found that the rate of decay increases with charging voltage and, also, for thinner films. Surface potential profiles were also measured, as was the current flowing through the sample during both charging and discharging. Additionally, numerical simulations were also developed to explore the physics that underpin the above observations. These imply that it is field distortion by the charge deposited on the sample surface which results in the development, during charging, of a non-uniform equilibrium surface potential profile, not lateral charge migration. The subsequent decay process is then determined by the initial distribution that forms and a combination of surface and bulk phenomena; bulk phenomena dominate.
\end{abstract}

\section{Introduction}

The decay of surface potential on insulating polymers has been studied extensively by many researchers [1-3], in connection with both fundamental and technological issues. From the materials perspective, most work has concentrated on highly insulating polymers, because their charge retention characteristics make them intrinsically suitable for electrostatic discharge studies or as a means of fabricating electrets. Fewer studies have reported on more conductive polymers, such as nylon or cellulose acetate. Indeed, to our knowledge, the only report of charge decay on the latter system was given by Kumar and Nath [4], which only considered the effect of surface potential and film thickness on the empirical decay process. The investigation described here was therefore undertaken to examine the underlying processes involved in the decay of surface potential decay on cellulose acetate. The motivation behind this choice of model material was the relatively rapid nature of the decay process and the well-known interaction of this polymer with water, particularly water vapour [3]. The objectives of the work were therefore to investigate surface potential decay as a function of key parameters, to examine current flows during the charging/discharging process and to develop a suitable numerical model of the process.

\section{Experimental}

Circular samples, $50 \mathrm{~mm}$ in diameter, were cut from cellulose acetate films, purchased from Agar Scientific Ltd. These were cleaned with demineralized water and sputtered gold electrodes were applied, as required. To study surface potential decay, the specimen was placed on a turntable and charged using a corona triode, with a needle of tip radius $0.1 \mathrm{~mm}$ and voltage $V_{a}$, and a grid of voltage $V_{g}$. For all the experiments reported here, the sample to grid $\left(d_{1}\right)$ and grid to anode $\left(d_{2}\right)$ separations were fixed at $16 \mathrm{~mm}$ and $25 \mathrm{~mm}$ respectively; $\left(V_{a}-V_{g}\right)$ was held at $-9 \mathrm{kV}$ throughout. A charging time of $20 \mathrm{~s}$ was found to be sufficient to ensure that the surface potential of the specimen, $V_{s}$, had reached its maximum. After charging the sample, the turntable could be manually rotated so as to move the specimen into position beneath the field meter (John Chubb Model JCI140), to enable $V_{s}$ to be recorded as a function of time. Alternatively, to observe spatial variations of surface potential, the turntable could be rotated by a DC motor at a constant angular velocity, such that the sample passed under the field meter periodically. This enabled the surface potential at different points along the diameter of the specimen to be recorded at periodic intervals during the charge decay process. In addition to the surface potential, associated current flows could also be measured, using a Keithley 485 picoammeter.

\section{Results}

\section{Decay of surface potential}

Typical surface potential decay plots are shown in Fig.1 for different values of $V_{g}$. From this, $V_{s}$ falls progressively with time; the initial rate increases with $V_{g}$ and drops as $V_{s}$ decays. An alternative representation of the same process is shown in Fig.2, which includes a sequence of surface potential profiles. Although the decrease in surface potential appears, initially, to be accompanied by a loss of charge from the edge of the specimen, thereafter, there is little to imply lateral charge migration. This behaviour is different from that which is commonly reported; equivalent traces for more insulating polymers [2,3] appear more rectangular in shape with higher peripheral charge densities. 


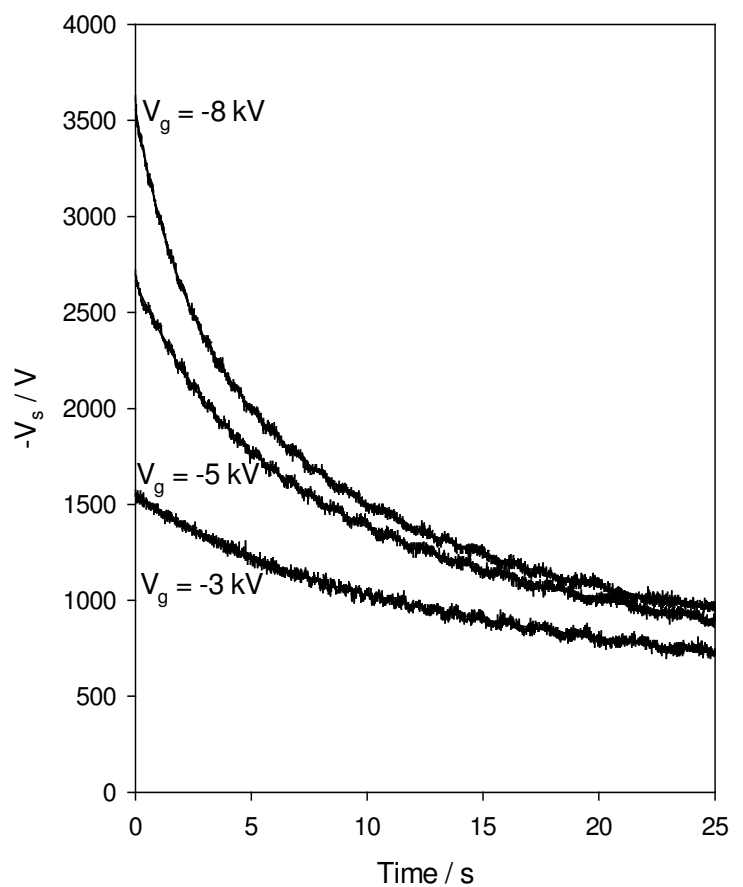

Figure1: Surface potential, $V_{s}$, as a function of time for different grid potentials, $V_{g} ; d_{l}=16 \mathrm{~mm}, d_{2}=25 \mathrm{~mm},\left(V_{a}-V_{g}\right)=-9 \mathrm{kV}$. Relative humidity $55 \%$; sample thickness $180 \mu \mathrm{m}$.

The temporal variation of the bulk $\left(i_{b}\right)$ and surface $\left(i_{s}\right)$ currents after charging were then investigated. The bulk current was determined by simply connecting the picoammeter to an electrode sputtered onto the reverse of the sample, while the lateral surface current was monitored via an additional, narrow annular electrode sputtered onto the periphery of the upper surface of the specimen. From Fig. $3, i_{s}$ decays simply with time, while the behaviour of $i_{b}$ is much more complex. The positive initial bulk current decays rapidly, reaching zero in about $0.2 \mathrm{~s}$, then changes its sign, and approaches zero. Finally, it again becomes slightly positive but, for reasons of scale, this cannot be seen in this figure. We explain this behaviour through a combination of processes relating to both free charges and polarization effects. The initial current, we suggest, is due to the free charges that already exist in or on the specimen, whilst the negative part is associated with depolarization. Similar behaviour has previously been reported for some other polymers [6], although, if the initial corona charging is large, the depolarization current may not be seen [5].

\section{Surface charging}

During charging, the potential at each point on the sample surface will rise towards the grid potential, until

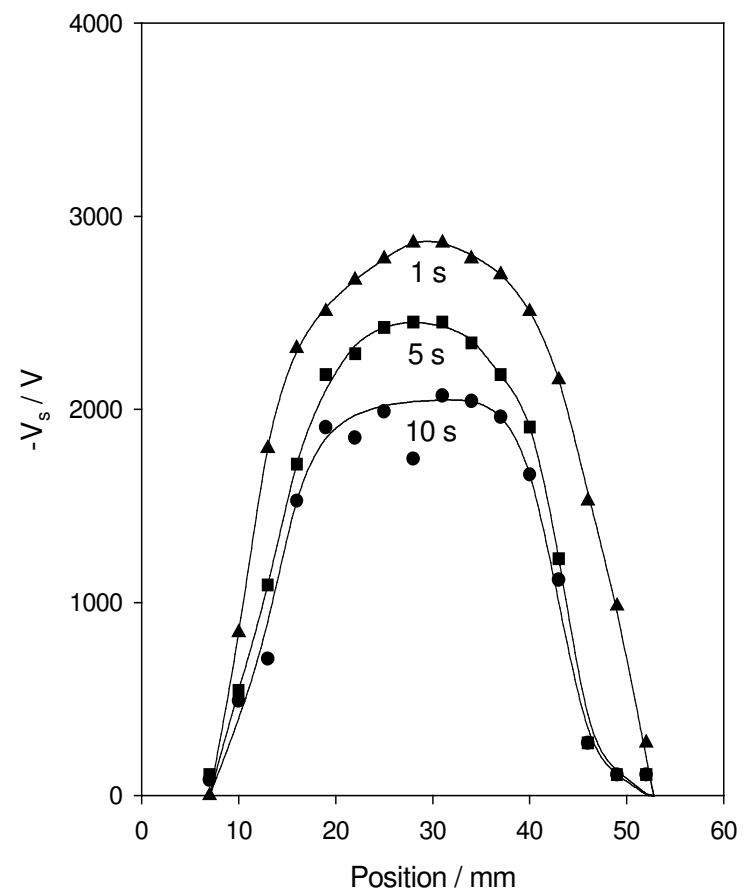

Figure 2: Decay of the surface potential profile with time; $V_{g}=-8 \mathrm{kV}, d_{l}=16 \mathrm{~mm}, d_{2}=25 \mathrm{~mm},\left(V_{a}-V_{g}\right)=-9 \mathrm{kV}$. Relative humidity $42 \%$; sample thickness $180 \mu \mathrm{m}$.

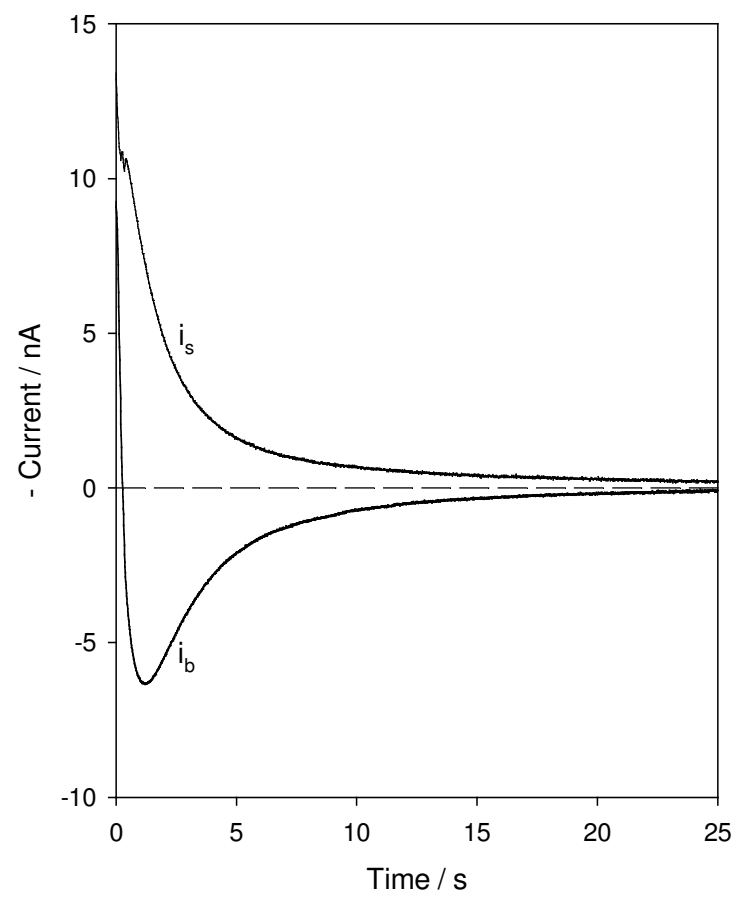

Figure 3: Variation of bulk, $i_{b}$, and surface, $i_{s}$, currents during surface potential decay; $V_{g}=-3.5 \mathrm{kV}, d_{1}=5 \mathrm{~mm}, d_{2}=25 \mathrm{~mm}\left(V_{a^{-}}\right.$ $\left.V_{g}\right)=-9 \mathrm{kV}$. Relative humidity $26 \%$, sample thickness $180 \mu \mathrm{m}$, 


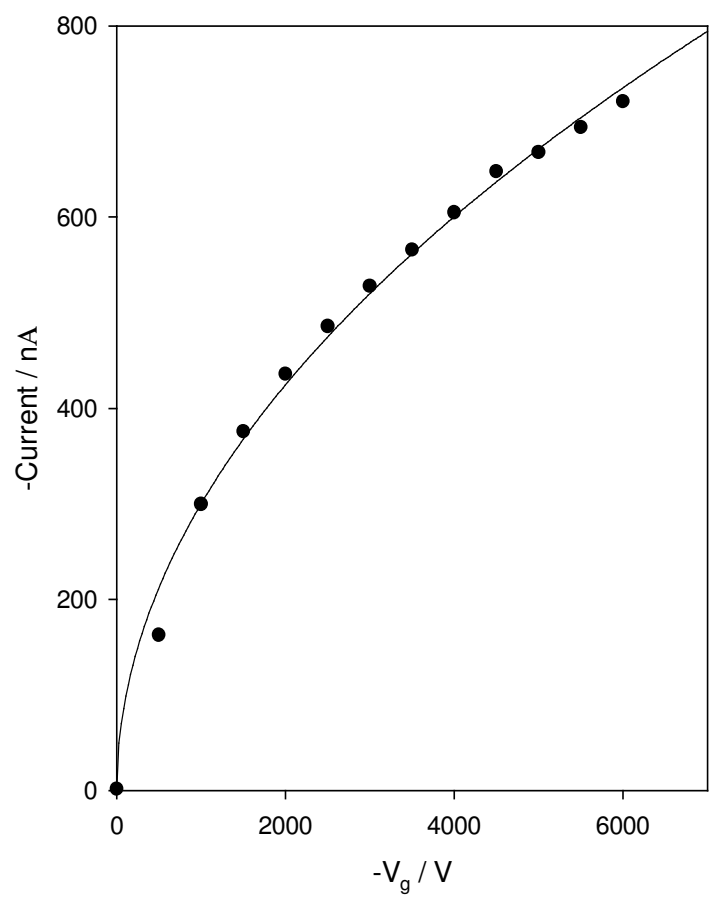

Figure 4: Effect of grid potential on the total current entering a grounded $50 \mathrm{~mm}$ diameter electrode. Here, $V_{g}$ corresponds to the potential difference between the grid and collector.

a local dynamic equilibrium is reached. However, before considering the currents flowing from our cellulose acetate sample, we will first consider the current density produced by the charging system. Figure 4 shows a plot of the total current entering a $50 \mathrm{~mm}$ diameter metal collector electrode. For comparison, the charging current flowing through the sample is shown in Fig.5. These were acquired under the same conditions of $d_{l}, d_{2}$ and $\left(V_{a}-V_{g}\right)$ as used for Fig.4 and with electrodes applied to the sample as for Fig.3. Here, the peripheral annular electrode was connected to ground and screened, to prevent the charging current from being swept directly into the sample's back electrode. From Fig.5, the total current flowing through the sample is $\sim 40 \mathrm{nA}$, when $V_{g}=-3.5 \mathrm{kV}$ whereas, from Fig.3, the initial value of bulk current is $\sim 10 \mathrm{nA}$. This discrepancy is due to two factors: Fig. 4 shows steady-state data whereas, in Fig.3, there was a small time delay between the end of charging and the commencement of measurements $(\sim 0.5 \mathrm{~s})$; the data shown in Fig.3 were obtained under very low humidity conditions, where the material conductivity is reduced. If the sample is considered to be a thin disc of diameter $5 \mathrm{~cm}$ and thickness $180 \mu \mathrm{m}$ then the volume and bulk currents can be estimated. The lowest available reliable conductivity data for cellulose acetate correspond to an applied voltage of $-2.7 \mathrm{kV}$. Then, the volume conductivity of

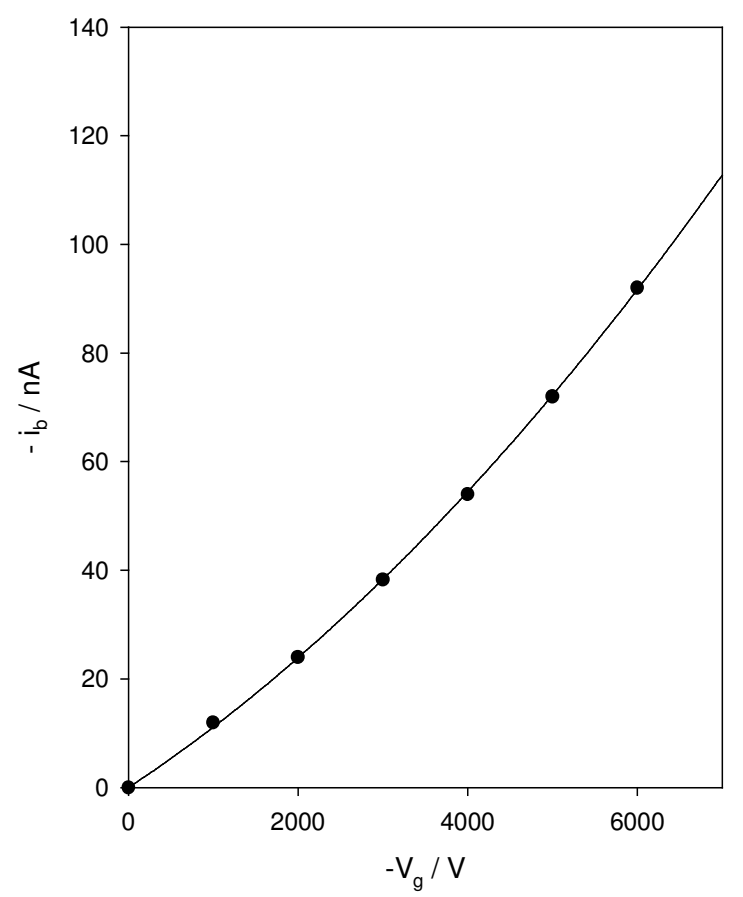

Figure 5: Plot of steady state bulk current, $i_{b}$, as a function of grid potential, $V_{g}$, with $\left(V_{a}-V_{g}\right)=-9 \mathrm{kV}$; outer annular electrode earthed and screened. Sample thickness $180 \mu \mathrm{m}$.

$\sim 1.6 \times 10^{-12} \mathrm{Sm}^{-1}$ [5] leads to a total bulk current of $\sim 45 \mathrm{nA}$ and the surface conductivity of $\sim 3.9 \times 10^{-15} \mathrm{~S}$ [5] gives a leakage current to earth across the sample's cylindrical, curved, vertical surface of $\sim 10 \mathrm{nA}$.

The purpose of the above is to demonstrate that our current data are both internally self-consistent and consistent with the conductivities of cellulose acetate. Also, and of more importance, they show that cellulose acetate is a material in which the initial surface and bulk discharge currents are of comparable magnitude, provided the surface is charged to a uniform potential. However, the above current values are all very much less than the flux from the charging system. If the surface potential $V_{s}=-2.7 \mathrm{kV}, V_{g}=-5 \mathrm{kV}$ (from Fig.1), the grid/sample potential difference is $2.3 \mathrm{kV}$ and the integrated charging flux over the area of the sample would be $\sim 500 \mathrm{nA}$. This difference is significant.

Although, by definition, the equilibrium surface potential corresponds to the condition where the current flowing into the sample equals the current flowing out of it, the equilibrium current emerging from the sample differs greatly from the charging current flux integrated over the sample area. To explore the evolution of surface potential in a circular thin film sample, a simple finite difference model was set up within MS Excel. This was based upon just the continuity equation, and considered the current flows across the surface into and 


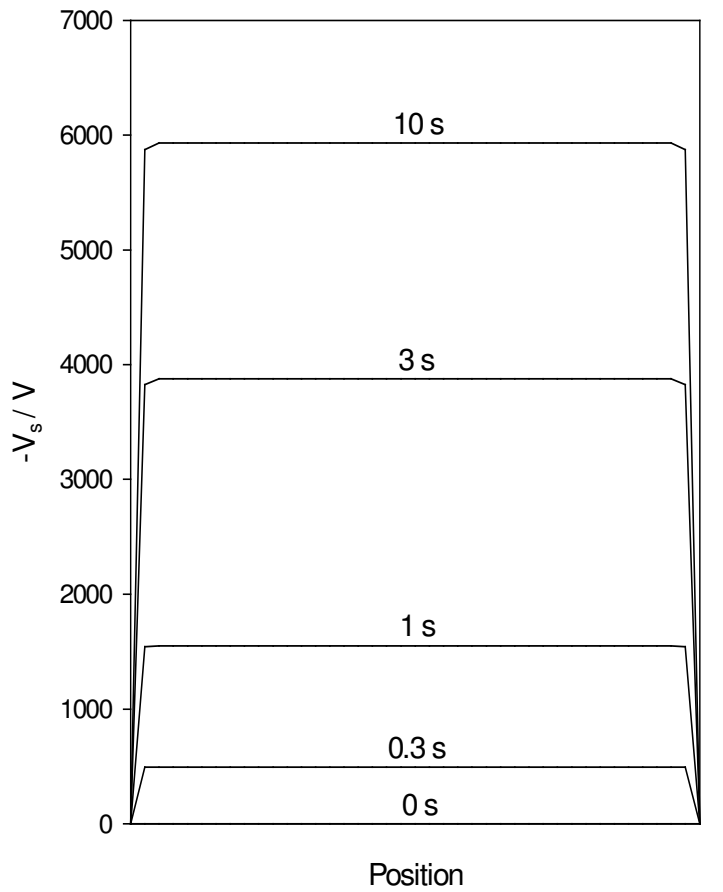

Figure 6: Simulated surface potential profile plots at different times during sample charging; grid potential $-6 \mathrm{kV}$, material parameters appropriate for $42 \%$ relative humidity.

out of each element, the current flowing through the bulk and the charging current, based upon the area of each element and the known dependence of flux density on the potential between the grid and a grounded electrode (ie Fig.4). Figure 6 shows the results of such a simulation. The explanation for the marked difference between both the absolute surface potential (cf Fig.1) that develops and the potential profile (cf Fig.2) is, we believe, field distortion at the sample periphery. As the sample's surface potential increases above that of the surrounding plane, the equipotentials become distorted, particularly near its periphery. The result of this will be two-fold.

(i) The charging current incident on the sample will be reduced, hence the measured $i_{b}$ values are very much less than would be anticipated from the charging flux.

(ii) The flux density will be non-uniform across the specimen, which will generate a non-uniform surface potential distribution. In particular, both will be reduced near the sample's edge

\section{Conclusions}

From the above data the charge decay process in cellulose acetate can be described in terms of a number of steps. Initially, the potential difference between the sample and the grid is large and uniform such that the rate of deposition of charge will be high. Some of this is lost through bulk conduction and edge leakage but, since the potential difference between the sample surface and the grounded backing electrode is, initially, low at all points, the surface potential grows rapidly. The increase in surface potential reduces the charging current and increases the loss of charge, through bulk conduction at the centre, and by both bulk and surface conduction at the periphery. More importantly, the equipotentials between the sample and the grid become distorted as the surface potential rises, such that the charging current is reduced globally and, more importantly, varies locally. The final equilibrium potential distribution is then determined by the condition that, at each point, the charge entering the sample equates to that lost, locally, by a combination of bulk and surface conduction. The field distortion limits the total current available for charging and results in a non-uniform potential distribution. During charge decay, the initial surface potential distribution is reduced, primarily, through bulk effects involving a combination of the migration of free charges and dielectric relaxation processes. In cellulose acetate, bulk effects dominate when the surface potential distribution is not characterized by high field regions near the sample's periphery.

\section{References}

[1] M. Ieda, G. Sawa and U. Shinohara, "Decay of electric charges on polymeric films", Electr. Eng. Jap., vol.88, pp. 73-88, June, 1968.

[2] Y. Hori, "The lateral migration of surface charges on poly(methyl methacrylate) graft-copolymerized onto polypropylene films and its dependency on relative humidity", $J$. Electrostatics, vol.48 pp. 127-143, Jan. 2000.

[3] D. K. Das Gupta, "Electrical properties of surfaces of polymeric insulators", IEEE Trans. Elect. Ins., vol.27, pp. 909-923, Oct. 1992.

[4] A. Kumar and R. Nath, "Study of the surface potential decay characteristics in cellulose acetate films", J. Electrostatics, vol.14, pp. 201-208, May-Aug. 1983.

[5] E. Borzabadi, A. S. Vaughan and A. G. Bailey, to be published.

[6] D. K. Das Gupta, "Conduction mechanisms and high-field effect in synthetic insulating polymers", IEEE Trans. Diel. Electr. Insul., vol.4, pp. 149-156, Apr. 1997.

Author address: Alun S. Vaughan, ECS, Faraday Building, University of Southampton, Southampton SO17 1BJ, UK, Email: asv@ecs.soton.ac.uk 